\title{
表观遗传修饰在神经退行性变性疾病中 的作用研究进展
}

曲文政 ${ }^{1}$, 庄英粮 ${ }^{1,2}$, 李学坤 ${ }^{1,2}$

1. 浙江大学医学院附属儿童医院国家儿童健康与疾病临床医学研究中心国家儿童区域 医疗中心, 浙江杭州 310052

2. 浙江大学转化医学研究院, 浙江杭州 310029

［摘＼cjkstart要］ 表观遗传修饰对神经发育、神经干细胞命运决定和神经系统的生理功 能发挥具有重要的调节作用。异常的表观遗传修饰与阿尔茨海默病、帕金森病和 亨廷顿病等神经退行性变性疾病的发生和发展有密切关系: 异常升高的 DNA 甲基 化修饰抑制了一些修复基因的表达, 影响烹廷顿病进展; 阿尔茨海默病患者大脑中 $\mathrm{H} 3 \mathrm{~K} 27 \mathrm{ac}$ 和 H3K9ac 组蛋白修饰增加, 影响神经变性; RNA 甲基化修饰在阿尔茨海 默病和帕金森病两种疾病动物模型中呈现差异化的改变。因此, 表观遗传修饰可 能作为神经系统疾病的潜在治疗靶点。本文综述了表观遗传修饰参与神经退行性 变性疾病及其分子机制的最新研究进展。

[关键词] 表观遗传学; DNA 修饰; 组蛋白修饰; RNA 修饰; 阿尔茨海默病;帕金森 病; 亭廷顿病; 综述

[中图分类号 ］R394;R741［文献标志码］A

\section{The roles of epigenetic modifications in neurodegenerative diseases}

QU Wenzheng ${ }^{1}$, ZHUANG Yingliang ${ }^{1,2}$, LI Xuekun ${ }^{1,2}$ (1. Children's Hospital, Zhejiang University School of Medicine, National Clinical Research Center for Child Health, National Children's Regional Medical Center, Hangzhou 310052, China; 2. Institute of Translational Medicine, Zhejiang University, Hangzhou 310029, China)

Corresponding author: LI Xuekun, E-mail: xuekun_li@zju.edu.cn, https://orcid.org/00000002-6985-6363

[ Abstract ] In neuronal system, epigenetic modifications are essential for neuronal development, the fate determination of neural stem cells and neuronal function. The

收稿日期: 2021-06-04 接受日期: 2021-08-30 网络预发表日期:2021-10-15

基金项目:国际科技合作重点项目计划(YS2017YFGH001214),国家自然科学基金(92049108)

第一作者:曲文政,助理实验师, 主要从事表观遗传修饰和神经系统疾病研究;E-mail:quwz@zju.edu.cn; https://orcid.org/ 0000-0002-1150-295X

通信作者:李学坤,教授,主要从事表观遗传修饰与神经发育及神经系统疾病领域的研究;E-mail:xuekun_li@zju.edu.cn; https ://orcid.org/0000-0002-6985-6363 
dysfunction of epigenetic regulation is closely related to occurrence and development of neurodegenerative diseases, including Alzheimer's disease, Parkinson's disease, Huntington's disease. Abnormally elevated DNA methylation inhibits the expression of some DNA repair-related genes and affects the progression of Huntington's disease. In the brain of Alzheimer's disease patients, the levels of H3K27ac and H3K9ac histone modifications increased. In addition, the alteration of RNA methylation in animal models of Alzheimer's disease and Parkinson's disease showed discrepancy trends. Therefore, epigenetic modifications may serve as potential therapeutic targets for neurodegenerative diseases. Here, we summarize the recent progress of the roles of epigenetic modifications in neurodegenerative diseases.

[ Key words ] Epigenetics; DNA modification; Histone modification; RNA modification; Alzheimer's disease; Parkinson's disease; Huntington's disease; Review

[J Zhejiang Univ (Med Sci), 2021, 50(5): 642-650.]

[缩略语］阿尔茨海默病(Alzheimer's disease,AD); 帕金森病(Parkinson's disease, PD); 亭廷顿病(Huntington's disease,HD);DNA 甲基化酶(DNA methyltransferase, DNMT); S-腺苷甲硫氨酸 (S-adenosylmethionine, SAM);5-甲基胞嘧啶 (5-methylcytosine , 5mC); 5-差烡基胞嘧啶 (5-hydroxymethylcytosine, 5hmC); $\beta$ 淀粉样蛋白 (amyloid $\beta$-protein, $\mathrm{A} \beta$ ); 肽基脯氨酰基顺反异构酶 (peptidyl-prolyl cis-trans isomerase, PPIase, PIN); $\alpha$ 突触核蛋白 ( $\alpha$-synuclein, SNCA); 亭廷顿蛋白 (huntingtin, HTT); 腺苷 $\mathrm{A}_{2 \mathrm{~A}}$ 受体 (adenosine $\mathrm{A}_{2 \mathrm{~A}}$ receptor, ADORA2A); 组蛋白 H3 第 27 位点赖氨酸乙酰化 (acetylated at lysine 27 of histone H3,H3K27ac); 组蛋白 H3 第9位点赖氨酸乙酰化 (acetylated at lysine 9 of histone H3, H3K9ac); 组蛋白脱乙酰酶 (histone deacetylase, HDAC); 组蛋白 $\mathrm{H} 3$ 第 4 位点赖氨酸三甲基化(trimethylated at lysine 4 of histone H3, H3K4me3); 富含 亮氨酸的重复激酶(leucine-rich repeat kinase, LRRK); 甲基转移酶样(methyltransferase like, METTL); 信使RNA(messenger RNA, mRNA); N6-甲基腺苷(N6-methylladenosine, $\mathrm{m}^{6} \mathrm{~A}$ )

表观遗传修饰是指不依赖于 DNA 序列改变 的基因表达水平的变化,主要包括DNA 修饰、组蛋 白修饰和 RNA 修饰(图 1)。研究表明, 表观遗传 修饰参与了包括胚胎发育、干细胞调控、细胞调 亡、器官稳态维持等多种生物学过程, 与多种疾病 的发生和发展关系密切 ${ }^{[1-3]}$ 。神经退行性变性疾 病是由于神经元的异常死亡导致的神经系统疾 病, 随着社会老龄化, 发病率逐渐升高。研究表 明, 表观遗传修饰与神经退行性变性疾病有着密 切关系 ${ }^{[4]}$ 。本文综述了表观遗传修饰在 AD、PD、 $\mathrm{HD}$ 等神经退行性变性疾病中的研究进展, 以期深 人认识不同表观遗传修饰类型在神经退行性变性 疾病中的作用, 为临床诊断与治疗提供新的 思路。

\section{DNA 修饰与神经退行性变性疾病}

DNA 甲基化修饰是在 DNMT 的催化作用下， 以 $\mathrm{SAM}$ 为甲基供体,将基因组中的胞嘧啶催化形 成 $5 \mathrm{mC}^{[5]}$ 。在正常组织中, $70 \%$ 90\%散在的 $\mathrm{CpG}$ 位点被甲基修饰，而高度富集 $\mathrm{CpG}$ 二核苷酸的 $\mathrm{CpG}$ 岛主要位于基因转录调控区附近并表现为非 甲基化状态。DNA 甲基化修饰可以抑制基因表 达, 在神经发育、神经功能调节、记忆的巩固和存 储等过程中发挥重要作用 ${ }^{[6-7]}$ 。

长期以来, DNA 甲基化被认为是一种稳定、 不可逆的修饰形式。2009年两项研究以及后续的 众多研究改变了这一观点, 进而拓展了DNA 修饰 的研究内容 ${ }^{[8-12]}$ 。研究发现, $5 \mathrm{mC}$ 可在 DNA 双加 


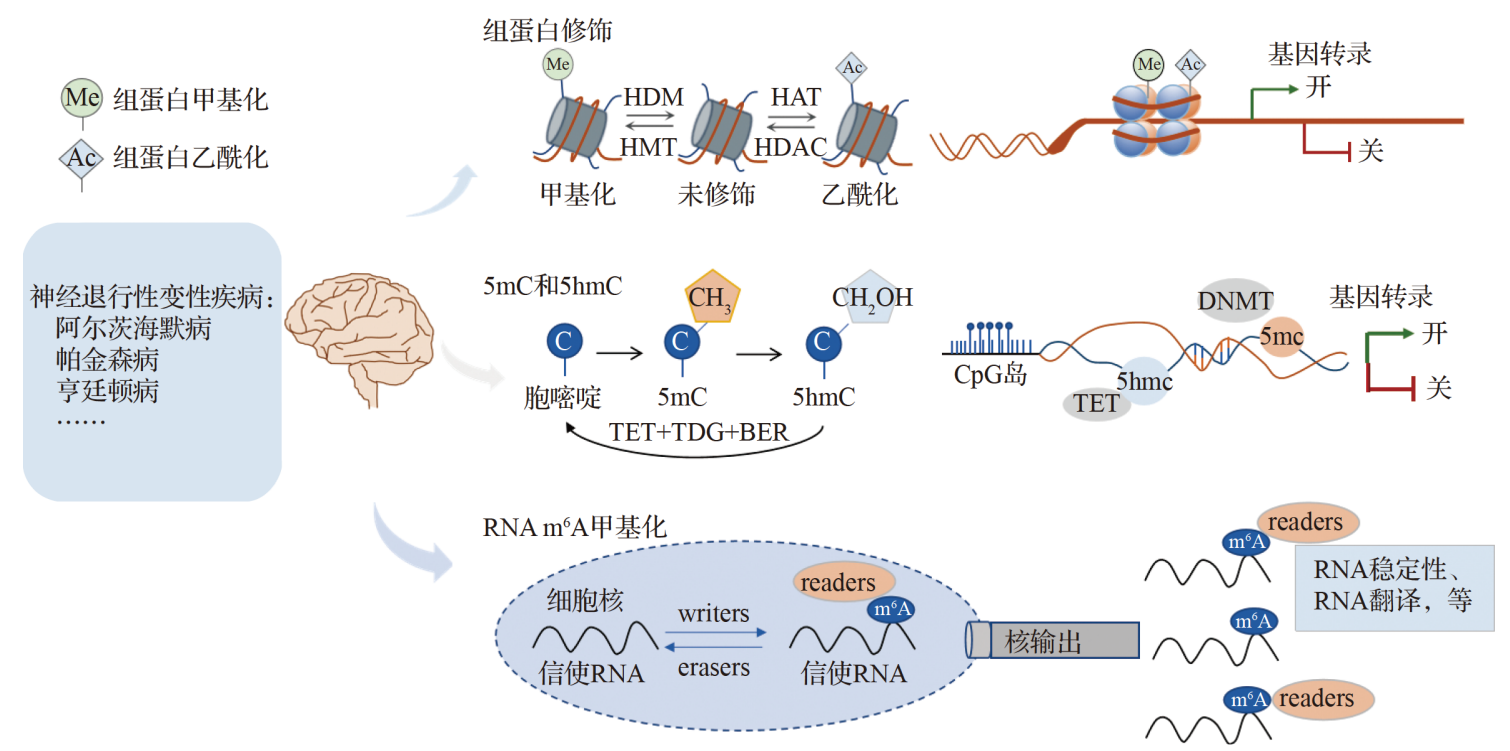

组蛋白甲基化由组蛋白甲基化酶(HMT)与组蛋白去甲基化酶(HDM)两大类酶调控, 组蛋白乙酰化由组蛋白乙酰化酶(HAT)和 组蛋白脱乙酰酶 (HDAC) 调控. 组蛋白甲基化或乙酰化修饰异常影响基因的转录表达. DNA 甲基化修饰是胞嘧啶在DNA 甲基化酶 (DNMT)的作用下转化成5-甲基胞嘧啶 $(5 \mathrm{mC}$ ). DNA 5-羟甲基化修饰是在 $5 \mathrm{mC}$ 去甲基化过程中, 在TET作用下转化成5-羟甲基胞嘧啶 (5hmC). $5 \mathrm{hmC}$ 可在TET和胸腺嘧啶DNA糖基化酶 (TDG) 的作用下, 经碱基切除修复 (BER)转化成胞嘧啶. RNA N6-甲基腺苷 $\left(\mathrm{m}^{6} \mathrm{~A}\right.$ )甲 基化由 “writers”、“erasers” 和 “readers” 蛋白调控, 其中 “writers” 是RNA甲基化酶,包括甲基转移酶样(METTL)3和METTL14 等, “erasers” 是RNA去甲基化酶,包括FTO和ALKBH5, “readers”包括YTHDF $1 / 2 / 3$ 等,调节RNA转运、剪切、翻译等.

图 1 组蛋白乙酰化与甲基化修饰、DNA修饰和RNA m ${ }^{6} \mathrm{~A}$ 甲基化修饰示意图

Figure 1 Diagram of epigenetic modifications including histone acetylation and methylation, DNA methylation, RNA M ${ }^{6} \mathrm{~A}$

氧酶TET(ten-eleven translocation)的作用下被氧化 为 $5 \mathrm{hmC} \mathrm{C}^{[8-9]}$ 。在哺乳动物大脑发育和衰老过程 中,DNA 5-着甲基化修饰呈现出一种动态特征, 在 海马颗粒神经元和小脑浦肯野细胞中高度富 集 ${ }^{[13-14]}$ 。进一步研究发现, $5 \mathrm{hmC}$ 可作为一种稳定 的表观遗传标志物存在,与神经发育、衰老、疾病 关系密切 ${ }^{[14-16]}$ 。DNA 甲基化通过调控基因表达， 影响突触可塑性以及记忆的形成、巩固和储存 ${ }^{[6]}$ 。

TET1 可通过影响 DNA 甲基化水平调节记忆的形 成、成年小鼠海马神经发生和认知功能 ${ }^{[17-18]}$ 。 $T E T 1$ 敲低会导致小鼠海马区神经元再生受损, 学 习、记忆能力及突触可塑性出现异常 ${ }^{[18]}$ 。TET2敲 除的小鼠出现成体神经发生和认知功能的异 常 ${ }^{[19]}$ 。TET3 也可调控突触传递功能 ${ }^{[20]}$, 小鼠下 丘脑前额叶皮层的 TET3 缺失会减弱条件恐惧记 忆的消退 ${ }^{[13]}$ 。

\subsection{DNA 修饰与 $\mathrm{AD}$}

$\mathrm{AD}$ 是一种最为常见的神经系统退行性疾病, 其临床特征主要表现为渐进性的记忆丧失以及认 知损伤, 病理学改变主要是脑内的 $A \beta$ 异常沉积和 $\tau$ 蛋白异常磷酸化所形成的神经元纤维缠结 ${ }^{[21]}$ 。

DNA 甲基化可以调控基因表达, 在 65 岁以上
$\mathrm{AD}$ 患者中 DNMT(如DNMT1)表达水平增加, DNA 甲基化水平增加,与 $\mathrm{AD}$ 风险基因 $A P O E \varepsilon 4$ 的表达 存在相关性 ${ }^{[22]}$ 。 $\mathrm{AD}$ 患者存在显著认知轨迹改变, 但传统的神经病理学对此只能进行部分解释。 Hüls 等 ${ }^{[23]}$ 对 636 例患者进行了一项大脑DNA 甲基 化与认知轨迹改变的关联研究, 发现编码血脑屏 障的一种重要蛋白质 CLDN5 的 DNA 甲基化修饰 异常, 提示 $C L D N 5$ 甲基化修饰异常引起的血脑屏 障功能障碍在 $\mathrm{AD}$ 患者早期认知能力下降中可能 发挥重要作用。PIN1 是一种丙基顺反异构酶,与 $\mathrm{AD}$ 的发病时间和其他病理特征有关。 $\mathrm{Ma}$ 等 ${ }^{[24]}$ 分 析了 80 例 $\mathrm{AD}$ 患者和 180 名健康者的 PINI 基因表 达和甲基化水平,发现PIN 1 甲基化与基因表达呈 显著负相关,而PIN1甲基化水平升高增加了 $\mathrm{AD}$ 的 发病风险。上述研究结果表明,DNA 甲基化修饰 的异常与 $\mathrm{AD}$ 的发生和发展存在密切关系。

目前研究表明, $5 \mathrm{hmC}$ 修饰在 $\mathrm{AD}$ 发生和发展 中也发挥了重要作用。AD 小鼠和 $\mathrm{AD}$ 患者病变组 织的研究结果均显示, 随着 $\mathrm{AD}$ 病程的进展, $5 \mathrm{hmC}$ 整体水平增加, 差异化 $5 \mathrm{hmC}$ 修饰主要位于外显子 区、内含子区和基因间区 ${ }^{[25-26]}$, 差异化 $5 \mathrm{hmC}$ 修饰 影响了与神经投射发育和神经发生相关基因的表 
达 $^{[26]}$ 。TET2敲除显著降低了 $5 \mathrm{hmC}$ 水平, 同时增 加了 $\mathrm{AD}$ 的严重程度, 而在正常衰老小鼠过表达 TET2 可以改善认知功能 ${ }^{[25,27]}$ 。 AD 小鼠神经元全 基因组水平 $5 \mathrm{hmC}$ 减少,过表达 TET家族成员中的 TET 催化域可以显著减弱神经变性过程,包括减 少 $\mathrm{A} \beta$ 积累和 $\tau$ 蛋白过磷酸化, 并改善 $\mathrm{AD}$ 小鼠大脑 突触功能障碍 ${ }^{[28]}$, 表明 $5 \mathrm{hmC}$ 失调在 $\mathrm{AD}$ 神经退行 性病变过程中发挥关键作用。

类脑器官 (brain organoid) 是一种研究大脑发 育和神经系统疾病的新兴模型。利用家族性 AD 患者诱导多能干细胞培养产生的类脑器官观察到 了 $\mathrm{AD}$ 的某些关键性病理特征, 如 $\tau$ 蛋白的过度磷 酸化和 $\mathrm{A} \beta$ 的聚集 ${ }^{[29]}$ 。转录组测序和 $5 \mathrm{hmC}$ 全基 因组图谱分析发现 $5 \mathrm{hmC}$ 修饰与基因表达呈显著 正相关, 鉴定了 25000 多个 $5 \mathrm{hmC}$ 差异化修饰位 点, 这些差异化修饰位点与 1000 多个表达改变的 基因相关联, $5 \mathrm{hmC}$ 修饰和转录水平同时升高的基 因功能与神经发育过程相关 ${ }^{[29]}$ 。该研究结果进 一步证实了 $\mathrm{AD}$ 发生和发展过程中 $5 \mathrm{hmC}$ 修饰的 改变。

\subsection{DNA 修饰与PD}

$\mathrm{PD}$ 是发病率仅次于 $\mathrm{AD}$ 的神经退行性变性疾 病,在 65 岁以上人群中的发病率高达 $1 \%$ 2\%。PD 的主要病理学改变是在神经元的细胞质中存在大 量 SNCA 聚集和黑质致密部多巴胺能神经元的死 $亡^{[30]}$ 。研究发现, DNA 甲基化水平在PD患者大 脑皮层中明显增加 ${ }^{[31]}$ 。PD最重要的风险基因之 $-S N C A$ 编码 $\mathrm{SNCA}, S N C A$ 表达水平升高与 PD 发病 有关, 而维持神经元功能需要正常表达水平的 $S N C A$ 。 $S N C A$ 甲基化抑制其表达, SNCA 的 1 号内 含子 $\mathrm{CpG}$ 岛低甲基化会导致 $\mathrm{SNCA}$ 表达水平增加, 进而引起 PD 症状 ${ }^{[32-33]}$ 。

鉴于SNCA 在PD 中的重要作用, 研究者针对 SNCA 的表达调控开展了 PD 相关治疗研究。左旋 多巴可以增加 $S N C A$ 启动子的甲基化水平, 从而降 低 $S N C A$ 的表达 ${ }^{[34]}$ 。基于 CRISPR/dCas9 与 DNMT3A 的催化域融合手段, 利用慢病毒载体靶 向调控SNCA 1 号内含子的甲基化, 结果显示由于 载体靶向该内含子上DNA 甲基化, 导致了 PD 患者 来源的神经元 $S N C A \mathrm{mRNA}$ 及其蛋白的下调, 减轻 了疾病相关的细胞表型特征 (如线粒体活性氧的 产生和细胞活性 $)^{[35]}$ 。上述研究表明, PD 患者存 在异常的 DNA 甲基化修饰, 并有可能成为一种 PD
早期诊断和治疗策略。

利用 MPTP (1-甲基-4-苯基-1,2,3,6-四氢吡 啶)或其活性产物 $\mathrm{MPP}^{+}$分别制备 $\mathrm{PD}$ 小鼠模型和细 胞模型, 体内研究发现黑质酪氨酸差岱化酶神经元 中 TET2水平显著升高, 体外细胞中检测到 $C d k n 2 A$ 和 $C d k l 5$ 启动子区域 $5 \mathrm{hmC}$ 修饰增加, $T E T 2$ 敲低可 以挽救细胞损伤和细胞周期阻滞 ${ }^{[36]}$ 。对 PD 患者 前额叶神经元进行 $5 \mathrm{mC}$ 和 $5 \mathrm{hmC}$ 测序发现, PD 患 者神经元中增强子有 1799 个差异甲基化修饰位 点, 其中超过 $70 \%$ 的位点甲基化水平升高, 涉及 1482 个启动子和 2885 个基因,包括TET2基因和已 知的 PD 风险基因, 如 DCTN1、PRKN、DJ-1等 ${ }^{[37]}$ 。 而且, TET2 失活能够减轻大脑炎症反应, 对炎症 引起的小鼠黑质多巴胺能神经元变性具有保护 作用 ${ }^{[37]}$ 。

\subsection{DNA 修饰与HD}

HD 是一种常染色体显性遗传的神经系统疾 病, 典型特征是舞蹈样症状,并伴有认知退化和精 神障碍。 $\mathrm{HD}$ 的致病基因为 $H T T$, 其第一个外显子 中三联密码子 $\mathrm{CAG}$ 的异常扩增导致 HD 发病。研 究发现, HD 小鼠的纹状体组织中DNA 甲基化发生 改变, 特别是与神经发育相关基因,包括 $A p-1$ 、 Sox 2、Pax6等启动子区域出现过度的甲基化修饰， 提示变异的 HTT 蛋白可能通过影响染色质结构而 影响神经发育过程中的基因表达,进而产生 HD 表 型 ${ }^{[38-39]}$ 。另外, 异常升高的 DNA 甲基化抑制了 DNA 修复基因的表达,并增加了异常扩增 $\mathrm{CAG}$ 结 构的不稳定性 ${ }^{[40]}$ 。与正常小鼠相比, Y AC128 HD 小鼠的纹状体和皮层 $5 \mathrm{hmC}$ 水平显著下降,差异化 $5 \mathrm{hmC}$ 修饰位点相关基因与神经发育和神经系统 功能存在密切的关系 ${ }^{[41]}$ 。纹状体氨基丁酸能神 经元中高水平表达 $A D O R A 2 A$, 该类型神经元在 $\mathrm{HD}$ 病变中最易受损。 $\mathrm{HD}$ 患者纹状体组织 $A D O R A 2 A$ 表达下降,研究发现 $A D O R A 2 A$ 基因 5'UTR 区域 $5 \mathrm{mC}$ 修饰增加, 而 $5 \mathrm{hmC}$ 修饰减少 ${ }^{[42]}$ 。

\section{2 组蛋白修饰与神经退行性变性疾病}

组蛋白是由 H2A、H2B、H3 和 H4 共同组成的 八聚体,其氨基端残基的结构比较松散,是翻译后 共价修饰的主要位点,包括乙酰化、磷酸化、甲基 化、泛素化、类泛素化和ADP核糖基化,这些修饰 可以改变染色体的三级结构, 从而调控基因 表达 ${ }^{[43]}$ 。 


\section{1 组蛋白修饰与 $\mathrm{AD}$}

对 $\mathrm{AD}$ 患者脑组织内嗅皮层乙酰化修饰的测 序研究发现了 4000 多个差异乙酰化修饰位点, 相 关的基因包括 $A P P 、 P S E N 1 、 P S E N 2$ 和 $M A P T$ 等 ${ }^{[44]}$ 。Nativio 等 ${ }^{[45]}$ 整合分析了 $A D$ 患者及老年/ 年轻健康对照的大脑转录组学、蛋白质组学和表 观基因组学分析。RNA 测序结果显示, $\mathrm{AD}$ 患者大 脑中组蛋白乙酰转移酶相关基因表达上调, $\mathrm{H} 3 \mathrm{~K} 27 \mathrm{ac}$ 和 $\mathrm{H} 3 \mathrm{~K} 9 \mathrm{ac}$ 修饰增加。在 AD 果蝇模型 中, H3K27ac 和 $H 3 \mathrm{~K} 9 \mathrm{ac}$ 修饰增加可加重 $A \beta_{42}$ 驱动 的神经变性 ${ }^{[45]}$ 。HDAC6 的抑制剂 CKD-504不仅 在 $\mathrm{AD}$ 动物模型的大脑中显著改变 $\tau$ 的互作蛋白谱 和降解病理性 $\tau$ 蛋白, 而且在 $\mathrm{AD}$ 患者来源的类脑 器官中也表现出类似的作用 ${ }^{[46]}$ 。HDAC3 的抑制 剂可以抑制 $\tau$ 磷酸化和乙酰化, 减少 $\mathrm{A} \beta$ 聚集, 改善 模型小鼠的认知功能 ${ }^{[47]}$ 。这些研究表明, 组蛋白 修饰特别是 H3K27ac 和 H3K9ac 可能作为 AD 治疗 的潜在表观遗传学靶点。

H3K4me3 修饰一般富集在活跃表达基因的启 动子。利用免疫组织化学法发现 $\mathrm{AD}$ 脑组织细胞 核内 $\mathrm{H} 3 \mathrm{~K} 4 \mathrm{me} 3$ 修饰水平降低, 有趣的是该研究在 细胞质中检测到 H3K4me3 修饰 ${ }^{[48]}$ 。 H $3 \mathrm{~K} 4 \mathrm{me} 3$ 修饰增加对应的基因与免疫反应密切相关; 而 H3K4me3 修饰下降对应的基因与突触功能和学习 记忆密切相关 ${ }^{[49]}$ 。需要指出的是, $\mathrm{AD}$ 不同脑区多 种组蛋白修饰标志物的研究结果存在差异, 这种差 异一方面可能是由 $\mathrm{AD}$ 亚型、疾病阶段和实验方法 引起,另一方面也反映了 $\mathrm{AD}$ 的复杂性。

\section{2 组蛋白修饰与 PD}

研究发现,PD 患者中脑多巴胺能神经元中的 组蛋白乙酰化水平显著升高 ${ }^{[50]}$ 。HDAC 抑制剂 HGC 可以保护多巴胺能神经元免受 $\mathrm{MPP}^{+}$诱导的 损伤, 改善 PD 小鼠的行为缺陷,维持线粒体的完 整性及其功能 ${ }^{[51]}$ 。用HGC处理SH-SY5Y 细胞 (人 神经母细胞瘤细胞)后进行蛋白质谱检测, 结果显 示 HGC 增加了 NADH 脱氢酶黄素蛋白 1 赖氨酸 28 位点的乙酰化修饰 ${ }^{[51]}$ 。研究发现, PD 患者的穿刺 活检标本中 $S N C A$ 启动子的 $\mathrm{H} 3 \mathrm{~K} 4 \mathrm{me} 3$ 显著增 加 ${ }^{[52]}$ 。为了解 $\mathrm{H} 3 \mathrm{~K} 4 \mathrm{me} 3$ 在 $\alpha$ 突触蛋白调控中的重 要性, 开发了基于 CRISPR/dCas9 的位点特异性 H3 K4me3 去甲基化系统, 该系统将去甲基化酶 $\mathrm{JARID} 1 \mathrm{~A}$ 催化域招募到 $S N C A$ 启动子, 显著降低了 $S N C A$ 启动子的 $\mathrm{H} 3 \mathrm{~K} 4 \mathrm{me} 3$ 修饰水平, 同时显著降低
了 $\alpha$ 突触蛋白的水平 ${ }^{[52]}$ 。

LRRK2 具有 GTP 酶和激酶活性, LRRK2突变 是常染色体显性家族性 PD 的主要原因。研究发 现, LRRK2 与 HDAC3 的 ser424位点结合并直接磷 酸化, 从而刺激 HDAC 活性, 促进组蛋白 H4 去乙 酰化, 导致基因转录抑制。对 $L r r k 2$ 敲除 $\left(\operatorname{Lrrk}^{-/}\right)$ 和正常 $\left(\operatorname{Lrrk} 2^{+/+}\right) 2$ 月龄、12 月龄小鼠分离的纹状 体组织进行转录组测序发现, Lrrk2缺乏加速了棘 状投射神经元的核肥大,并诱导了树突萎缩、体细 胞肥大和核内陷 ${ }^{[53]}$ 。此外, 在 $L r r k 2^{-1-}$ 老年小鼠纹 状体神经元中还观察到核DNA 损伤增加和组蛋白 甲基化异常, 以及参与调节神经元兴奋性、基因组 稳定性和蛋白质稳态的分子通路的改变 ${ }^{[53]}$, 表明 LRRK2 的致病作用与组蛋白修饰存在密切关系。

\section{3 组蛋白修饰与 HD}

转录失调是 HD 的一个关键特征。HD 患者 体细胞 $\mathrm{CAG}$ 重复的增加影响致病性过程的速率, 最终导致神经元死亡, 转录失调可能是其中一个 原因。为了识别在 $\mathrm{CAG}$ 扩增和/或下游致病过程 中起作用的修饰因子, 研究人员在 HD 小鼠中测试 了基因敲除 $H d a c 2$ 或 $H d a c 3$ 对 CAG 扩增和纹状体神 经元的影响。转录组测序数据表明,两种敲除均 减弱了 $\mathrm{CAG}$ 的扩增, Hdac2 敲除降低了 HTT蛋白的 病理学影响,影响了基因表达 ${ }^{[54]}$ 。研究结果提示 突变 $H t t$ 等位基因与 $H d a c 2$ 之间存在复杂关系, 为 靶向 HD 的转录失调治疗提供了理论依据。

对 II 级 HD 患者和对照组的纹状体 (如尾状 核)进行 H3K27ac 染色质免疫共沉淀测序发现,HD 患者纹状体中 $\mathrm{H} 3 \mathrm{~K} 27 \mathrm{ac}$ 水平降低的基因与神经元 活动和功能相关 ${ }^{[5]}$ 。Yildirim 等 ${ }^{[56]}$ 发现 HD 小鼠 在症状前阶段的纹状体中存在转录异常和组蛋白 H3K27 乙酰化变化。通过对测序数据整合分析, 确 定了Elk-1 转录因子作为 HD 前驱症状变化的候选 调节因子。外源表达Elk-1 缓解了 HD 小鼠的转录 失调, 对 HD 原代纹状体细胞发挥保护作用, 提示 Elk-1 可作为缓解HD病理学改变的潜在靶点 ${ }^{[56]}$ 。

\section{RNA 甲基化修饰与神经退行性变性疾病}

迄今, 在哺乳动物 $\mathrm{mRNA}$ 、转运 RNA、核糖体 RNA 及其他非编码RNA上发现了超过 100 种的修 饰方式 ${ }^{[57]}$ 。其中, $m R N A$ 的主要修饰有 $\mathrm{m}^{6} \mathrm{~A} 、 5$-甲 基胞苷、 $N^{7}$-甲基鸟苷、 $2^{\prime}-O$-甲基化核苷、 $N^{6}$-羟甲 基腺苷和 $N^{6}$-甲酰基腺苷等修饰。其中, $\mathrm{m}^{6} \mathrm{~A}$ 甲基 
化是真核生物中最普遍的修饰。高通量测序揭示 $了 \mathrm{~m}^{6} \mathrm{~A}$ 的以下特征: (1)具有组织特异性, 在脑内含 量最为丰富; (2)不仅存在于 $m R N A$, 还存在于长链 非编码 RNA, 以及微 RNA; (3) $\mathrm{m}^{6} \mathrm{~A}$ 并非出现在所有 $m R N A$; (4)主要富集区域序列是 $\mathrm{RRm}^{6} \mathrm{ACH}$ （R:嘌 呤; $\mathrm{H}$ : 非鸟嘌呤碱基 $)^{[58-59]}$ 。

$\mathrm{m}^{6} \mathrm{~A}$ 甲基化的生物学效应是由 “writers” (RNA 甲基化酶)、“erasers” (RNA 去甲基化酶)和 “readers” (阅读蛋白)介导的。RNA 甲基化酶复合 物包括 METTL3、METTL14等, 催化 mRNA的 $\mathrm{m}^{6} \mathrm{~A}$ 甲 基化 ${ }^{[60]}$ 。研究证实 $\mathrm{RNA}$ 去甲基化酶 $\mathrm{FTO}$ 和 ALKBH5 可以介导这种甲基化的可逆去除 ${ }^{[61-62]}$ 。 此外, YTH 结构域家族蛋白作为主要 $\mathrm{m}^{6} \mathrm{~A}$ 结合蛋 白调节 RNA 代谢, 包括 $\mathrm{mRNA}$ 剪切、降解和翻 译 ${ }^{[63]}$ 。目前证据提示, $\mathrm{m}^{6} \mathrm{~A}$ 修饰对 mRNA 的稳定 性、剪切、翻译、运输和细胞定位等发挥重要调控 作用 ${ }^{[63-65]} ; \mathrm{m}^{6} \mathrm{~A}$ 失调存在于癫㾁、抑郁症和神经退 行性变性疾病等人类神经系统疾病中 ${ }^{[66]}$ 。

\section{1 RNA m ${ }^{6} \mathrm{~A}$ 甲基化与 $\mathrm{AD}$}

研究发现, $m^{6} \mathrm{~A}$ 修饰在神经发育和衰老过程 中表现出时间和空间的动态变化 ${ }^{[67]} \circ \mathrm{m}^{6} \mathrm{~A}$ 具有明 显的组织特异性, 在下丘脑中最为明显。在小鼠 和人类衰老过程中明显观察到更多的 $\mathrm{m}^{6} \mathrm{~A}$ 位点。 差异 $\mathrm{m}^{6} \mathrm{~A}$ 位点富集在衰老相关的信号途径, 且与 mRNA 表达呈负相关 ${ }^{[67]}$ 。该研究表明,在 $\mathrm{AD}$ 小鼠 中, 与 $\mathrm{AD}$ 疾病进展相关转录本的 $\mathrm{m}^{6} \mathrm{~A}$ 甲基化水平 降低伴随着相应蛋白表达水平下降, 提示 $\mathrm{m}^{6} \mathrm{~A}$ 修 饰可能在衰老和神经退行性变性疾病中发挥重要 作用。

$\mathrm{AD}$ 患者认知障碍与大脑中 RNA 和蛋白质的 表达失调有关, 而 METTL3 在 AD 海马区的异常表 达和分布表明 $\mathrm{m}^{6} \mathrm{~A}$ 修饰可能与 $\mathrm{AD}$ 发病相关。对 $\mathrm{AD}$ 小鼠和正常小鼠的 $\mathrm{m}^{6} \mathrm{~A}$ 甲基化进行定量研究, 结果显示 $\mathrm{AD}$ 小鼠皮质和海马的 $\mathrm{m}^{6} \mathrm{~A}$ 修饰水平升 高。高通量测序结果显示, $\mathrm{m}^{6} \mathrm{~A}$ 修饰水平在 $\mathrm{AD}$ 小 鼠许多基因中升高, 并且 $\mathrm{m}^{6} \mathrm{~A}$ 甲基化酶 METTL3 表 达增加, 而 $\mathrm{m}^{6} \mathrm{~A}$ 去甲基化酶FTO 表达降低 ${ }^{[68]}$ 。上 述研究结果提示, $\mathrm{mRNA}$ 的 $\mathrm{m}^{6} \mathrm{~A}$ 修饰可能与 $\mathrm{AD}$ 的 发展有密切关系, 需要更深人的研究进一步验证。

\subsection{RNA m ${ }^{6} \mathrm{~A}$ 甲基化与 PD}

目前, 关于 $\mathrm{m}^{6} \mathrm{~A}$ 修饰在 PD 中作用研究较少。 有研究对 1647 例散发 PD 患者和1372名汉族健康 对照者进行了全面的 $\mathrm{m}^{6} \mathrm{~A}$ 修饰基因分析 ${ }^{[69]}$ 。根据
50 岁前或50岁后是否出现运动症状,将PD患者分 为早发期 $P D$ 患者和晚发期 PD 患者, 对罕见突变 进行基于基因负荷分析,对常见突变进行单变异 关联分析。结果表明,所有 $\mathrm{m}^{6} \mathrm{~A}$ 修饰相关基因 (10 个)中发现 214 个罕见突变,在 7 个基因中发现 16 个常见突变,但这些突变的关联分析结果并不显 著 ${ }^{[69]}$ 。也有研究发现, 在神经毒素 6-差基多巴胺 诱导的 PC12 细胞和 PD 大鼠脑纹状体中, mRNA 的 整体 $\mathrm{m}^{6} \mathrm{~A}$ 水平下调 ${ }^{[70]}$ 。过表达 $F T O$ 或 $\mathrm{m}^{6} \mathrm{~A}$ 抑制剂 均能降低多巴胺能神经元中 $\mathrm{m}^{6} \mathrm{~A}$ 修饰水平, $\mathrm{N}$-甲 基-D-天冬氨酸受体 1 的表达增加, 增加氧化应激 和钙离子内流, 导致细胞调亡 ${ }^{[70]}$ 。由于相关研究 资料较少, 关于 $\mathrm{m}^{6} \mathrm{~A}$ 修饰在 PD 中的具体作用仍有 待深人研究。

\section{4 结 语}

目前包括 $\mathrm{AD} 、 \mathrm{PD}$ 和 $\mathrm{HD}$ 在内的神经退行性变 性疾病对人类健康危害颇大, 由于其发病机制复 杂, 缺乏有效的治疗手段。随着研究的深人,越来 越多的证据表明表观遗传修饰的紊乱可能在神经 退行性变性疾病的进展过程中发挥了关键作用, 并可能作为潜在的治疗靶点。

开发有效的神经退行性变性疾病治疗技术需 要加深对疾病本身的认识。目前, 神经退行性变 性疾病的实验数据绝大多数是基于细胞和小动物 模型, 较少的数据来自于灵长类和人脑组织。未 来需要从灵长类等大动物模型中获得更多关于疾 病机制的数据。同时,采用新型的实验模型,如体 外培养三维类脑器官, 模拟神经退行过程和进行 药物研发对现有的研究很有帮助。

需要指出的是, 表观遗传修饰本身就具有相 当的复杂性,受到代谢(饮食)、环境等诸多因素的 影响, 需要谨慎解读其在神经退行性变性疾病中 的作用。譬如, 目前的研究结果显示 RNA 甲基化 修饰在 $\mathrm{AD}$ 和 PD 两种神经退行性变性疾病动物模 型中的变化呈现相反的趋势, 究竟这是实际情况 的反映还是实验误差导致需要深人研究。同时, 关于表观遗传学标志物作为神经退行性变性疾病 的靶标仍然需要更多的实验数据, 利用高效且特 异性的基因编辑技术治疗神经退行性变性疾病仍 然面临很多挑战。

利益冲突 所有作者均声明不存在利益冲突 


\section{参考文献}

[1] LI X, JIN P. Roles of small regulatory RNAs in determining neuronal identity $[\mathrm{J}]$. Nat Rev Neurosci, 2010, 11(5): 329-338.

[2] YAO B, CHRISTIAN K M, HE C, et al. Epigenetic mechanisms in neurogenesis $[\mathrm{J}]$. Nat Rev Neurosci, 2016, 17(9): 537-549.

[3] GREENBERG M V C, BOURC'HIS D. The diverse roles of DNA methylation in mammalian development and disease[J]. Nat Rev Mol Cell Biol, 2019, 20(10): 590-670.

[4] ARMSTRONG M J, JIN Y, ALLEN E G, et al. Diverse and dynamic DNA modifications in brain and diseases[J]. Hum Mol Genet, 2019, 28(R2): R241-R253.

[5] JONES P A. Functions of DNA methylation: islands, start sites, gene bodies and beyond $[\mathrm{J}]$. Nat Rev Genet, 2012, 13(7): 484-492.

[6] HALDER R, HENNION M, VIDAL R O, et al. DNA methylation changes in plasticity genes accompany the formation and maintenance of memory $[\mathrm{J}]$. Nat Neurosci, 2016, 19(1): 102-110.

[7] GREENBERG M, BOURC'HIS D. The diverse roles of DNA methylation in mammalian development and disease[J]. Nat Rev Mol Cell Biol, 2019, 20(10): 590-607.

[8] TAHILIANI M, KOH K P, SHEN Y, et al. Conversion of 5-methylcytosine to 5-hydroxymethylcytosine in mammalian DNA by MLL partner TET1[J]. Science, 2009, 324(5929): 930-935.

[9] KRIAUCIONIS S, HEINTZ N. The nuclear DNA base 5-hydroxymethylcytosine is present in Purkinje neurons and the brain $[\mathrm{J}]$. Science, 2009, 324(5929): 929-930.

[10] GU T P, GUO F, YANG H, et al. The role of Tet3 DNA dioxygenase in epigenetic reprogramming by oocytes $[\mathrm{J}]$. Nature, 2011, 477(7366): 606-610.

[11] ITO S, D’ALESSIO A C, TARANOVA O V, et al. Role of TET proteins in $5 \mathrm{mC}$ to $5 \mathrm{hmC}$ conversion, ES-cell self-renewal and inner cell mass specification $[\mathrm{J}]$. Nature, 2010, 466(7310): 1129-1133.

[12] KOHLI R M, ZHANG Y. TET enzymes, TDG and the dynamics of DNA demethylation[J]. Nature, 2013, 502(7472): 472-479.

[13] WU X, ZHANG Y. TET-mediated active DNA demethylation: mechanism, function and beyond $[\mathrm{J}]$. Nat Rev Genet, 2017, 18(9): 517-534.

[14] SZULWACH K E, LI X, LI Y, et al. 5-hmC-mediated epigenetic dynamics during postnatal neurodevelopment and aging $[\mathrm{J}]$. Nat Neurosci, 2011, 14(12): 1607-1616.

[15 ] LISTER R, MUKAMEL E A, NERY J R, et al. Global epigenomic reconfiguration during mammalian brain development $[$ J $]$. Science, 2013, 341(6146): 1237905.

[16] SUN W, ZANG L, SHU Q, et al. From development to diseases: the role of $5 \mathrm{hmC}$ in brain $[\mathrm{J}]$. Genomics, 2014, 104(5): 347-351.

[17] KAAS G A, ZHONG C, EASON D E, et al. TET1 controls CNS 5-methylcytosine hydroxylation, active DNA demethylation, gene transcription, and memory formation[J]. Neuron, 2013, 79(6): 1086-1093.

[18] ZHANG R R, CUI Q Y, MURAI K, et al. Tet1 regulates adult hippocampal neurogenesis and cognition $[J]$. Cell Stem Cell, 2013, 13(2): 237-245.

[19] LI X, YAO B, CHEN L, et al. Ten-eleven translocation 2 interacts with forkhead box $\mathrm{O} 3$ and regulates adult neurogenesis[J]. Nat Commun, 2017, 8(1): 15903.

[20 ] YU H, SU Y, SHIN J, et al. TET3 regulates synaptic transmission and homeostatic plasticity via DNA oxidation and repair $[\mathrm{J}]$. Nat Neurosci, 2015, 18(6): 836-843.

[21] GOASZEWSKA A, BIK W, MOTYL T, et al. Bridging the gap between Alzheimer's disease and Alzheimer's-like diseases in animals[J]. Int J Mol Sci, 2019, 20(7): 1664.

[22] DI FRANCESCO A, AROSIO B, FALCONI A, et al. Global changes in DNA methylation in Alzheimer's disease peripheral blood mononuclear cells $[\mathrm{J}]$. Brain Behav Immun, 2015, 45: 139-144.

[23] HÜLS A, ROBINS C, CONNEELY K N, et al. Brain DNA methylation patterns in CLDN5 associated with cognitive decline $[\mathrm{J}]$. Biol Psychiatry, 2021, online.

[24] MA S L, TANG N L S, LAM L C W. Promoter methylation and gene expression of Pin1 associated with the risk of Alzheimer's disease in southern Chinese[J]. Curr Alzheimer Res, 2021, 17(13): 1232-1237.

[25 ] SHU L, SUN W, LI L, et al. Genome-wide alteration of 5-hydroxymenthylcytosine in a mouse model of Alzheimer's disease $[\mathrm{J}]$. BMC Genomics, 2016, 17(1): 381 .

[26] BERNSTEIN A I, LIN Y, STREET R C, et al. 5Hydroxymethylation-associated epigenetic modifiers of Alzheimer's disease modulate tau-induced neurotoxicity [J]. Hum Mol Genet, 2016, 25(12): 2437-2450.

[27 ] GONTIER G, IYER M, SHEA J M, et al. TET2 rescues age-related regenerative decline and enhances cognitive function in the adult mouse brain $[\mathrm{J}]$. Cell Rep, 2018, 22(8): 1974-1981.

[28] ZHANG Y, ZHANG Z, LI L, et al. Selective loss of $5 \mathrm{hmC}$ promotes neurodegeneration in the mouse model of Alzheimer's disease[J]. FASEB J, 2020, 34(12): 16364-16382.

[29] KUEHNER J N, CHEN J, BRUGGEMAN E C, et al. 5hydroxymethylcytosine is dynamically regulated during forebrain organoid development and aberrantly 
altered in Alzheimer's disease $[\mathrm{J}]$. Cell Rep, 2021, 35(4): 109042.

[30] JANKOVIC J, TAN E K. Parkinson's disease: etiopathogenesis and treatment $[\mathrm{J}]$. J Neurol Neurosurg Psychiatry, 2020, 91(8): 795-808.

[31] KAUT O, KUCHELMEISTER K, MOEHL C, et al. 5methylcytosine and 5-hydroxymethylcytosine in brains of patients with multiple system atrophy and patients with Parkinson's disease $[\mathrm{J}]$. J Chem Neuroanat, 2019, 96: 41-48.

[32] MATSUMOTO L, TAKUMA H, TAMAOKA A, et al. CpG demethylation enhances alpha-synuclein expression and affects the pathogenesis of Parkinson's disease[J/OL]. PLoS One, 2010, 5(11): e15522.

[33] DESPLATS P, SPENCER B, COFFEE E, et al. $\alpha-$ synuclein sequesters Dnmt1 from the nucleus $[\mathbf{J}]$. J Biol Chem, 2011, 286(11): 9031-9037.

[34] SCHMITT I, KAUT O, KHAZNEH H, et al. L-dopa increases $\alpha$-synuclein DNA methylation in Parkinson's disease patients in vivo and in vitro $[\mathrm{J}]$. Mov Disord, 2015, 30(13): 1794-1801.

[35 ] KANTOR B, TAGLIAFIERRO L, GU J, et al. Downregulation of SNCA expression by targeted editing of DNA methylation: a potential strategy for precision therapy in $\mathrm{PD}[\mathrm{J}]$. Mol Ther, 2018, 26(11): 2638-2649.

[36] WU T T, LIU T, LI X, et al. TET2 - mediated $C d k n 2 A$ DNA hydroxymethylation in midbrain dopaminergic neuron injury of Parkinson's disease $[\mathrm{J}]$. Hum Mol Genet, 2020, 29(8): 1239-1252.

[37] MARSHALL L L, KILLINGER B A, ENSINK E, et al. Epigenomic analysis of Parkinson's disease neurons identifies TET2 loss as neuroprotective $[\mathrm{J}]$. Nat Neurosci, 2020, 23(10): 1203-1214.

[38] NG C W, YILDIRIM F, YAP Y S, et al. Extensive changes in DNA methylation are associated with expression of mutant huntingtin[J]. Proc Natl Acad Sci U S A, 2013, 110(6): 2354-2359.

[39] KERSCHBAMER E, BIAGIOLI M. Huntington's disease as neurodevelopmental disorder: altered chromatin regulation, coding, and non-coding RNA transcription [J]. Front Neurosci, 2015, 9: 509.

[40] MOLLICA P A, REID J A, OGLE R C, et al. DNA methylation leads to DNA repair gene down-regulation and trinucleotide repeat expansion in patient-derived huntington disease cells $[J]$. Am J Pathol, 2016, 186(7): 1967-1976.

[41] WANG F, YANG Y, LIN X, et al. Genome-wide loss of 5 -hmC is a novel epigenetic feature of Huntington's disease $[\mathrm{J}]$. Hum Mol Genet, 2013, 22(18): 3641-3653.

[42] VILLAR-MENÉNDEZ I, BLANCH M, TYEBJI S, et al. Increased 5-methylcytosine and decreased 5- hydroxymethylcytosine levels are associated with reduced striatal A2AR levels in Huntington's disease $[\mathrm{J}]$. Neuromolecular Med, 2013, 15(2): 295-309.

[43] JAKOVCEVSKI M, AKBARIAN S. Epigenetic mechanisms in neurological disease $[\mathrm{J}]$. Nat Med, 2012, 18(8): 1194-1204.

[44] MARZI S J, LEUNG S K, RIBARSKA T, et al. A histone acetylome-wide association study of Alzheimer's disease identifies disease-associated H3K27ac differences in the entorhinal cortex $[\mathrm{J}]$. Nat Neurosci, 2018, 21(11): 1618-1627.

[45] NATIVIO R, LAN Y, DONAHUE G, et al. An integrated multi-omics approach identifies epigenetic alterations associated with Alzheimer's disease $[\mathrm{J}]$. Nat Genet, 2020, 52(10): 1024-1035.

[46] CHOI H, KIM H J, YANG J, et al. Acetylation changes tau interactome to degrade tau in Alzheimer's disease animal and organoid models $[\mathrm{J} / \mathrm{OL}]$. Aging Cell, 2020, 19(1): e13081.

[47] JANCZURA K J, VOLMAR C H, SARTOR G C, et al. Inhibition of HDAC3 reverses Alzheimer's diseaserelated pathologies in vitro and in the $3 \mathrm{xTg}-\mathrm{AD}$ mouse model $[J / O L]$. Proc Natl Acad Sci U S A, 2018, 115(47): E11148-E11157.

[48] MASTROENI D, DELVAUX E, NOLZ J, et al. Aberrant intracellular localization of $\mathrm{H} 3 \mathrm{k} 4 \mathrm{me} 3$ demonstrates an early epigenetic phenomenon in Alzheimer's disease[J]. Neurobiol Aging, 2015, 36(12): 3121-3129.

[49] GJONESKA E, PFENNING A R, MATHYS H, et al. Conserved epigenomic signals in mice and humans reveal immune basis of Alzheimer's disease $[\mathrm{J}]$. Nature, 2015, 518(7539): 365-369.

[50] PARK G, TAN J, GARCIA G, et al. Regulation of histone acetylation by autophagy in Parkinson disease[J]. J Biol Chem, 2016, 291(7): 3531-3540.

[51] LI B, YANG Y, WANG Y, et al. Acetylation of NDUFV1 induced by a newly synthesized HDAC6 inhibitor HGC rescues dopaminergic neuron loss in Parkinson models [J]. iScience, 2021, 24(4): 102302.

[52] GUHATHAKURTA S, KIM J, ADAMS L, et al. Targeted attenuation of elevated histone marks at SNCA alleviates $\alpha$-synuclein in Parkinson's disease $[\mathrm{J} / \mathrm{OL}]$. EMBO Mol Med, 2021, 13(2): e12188.

[53 ] CHEN X, XIE C, TIAN W, et al. Parkinson's diseaserelated Leucine-rich repeat kinase 2 modulates nuclear morphology and genomic stability in striatal projection neurons during aging $[\mathrm{J}]$. Mol Neurodegener, 2020, 15(1): 12 .

[54] KOVALENKO M, ERDIN S, ANDREW M A, et al. Histone deacetylase knockouts modify transcription, CAG instability and nuclear pathology in Huntington 
disease mice[J/OL]. eLife, 2020, 9: e55911.

[55] MERIENNE N, MEUNIER C, SCHNEIDER A, et al. Cell-type-specific gene expression profiling in adult mouse brain reveals normal and disease-state signatures[J]. Cell Rep, 2019, 26(9): 2477-2493.e9.

[56] YILDIRIM F, NG C W, KAPPES V, et al. Early epigenomic and transcriptional changes reveal Elk-1 transcription factor as a therapeutic target in Huntington's disease[J]. Proc Natl Acad Sci U S A, 2019, 116(49): 24840-24851

[57 ] LI S, MASON C E. The pivotal regulatory landscape of RNA modifications $[J]$. Annu Rev Genom Hum Genet, 2014, 15(1): 127-150.

[58 ] MEYER K D, PATIL D P, ZHOU J, et al. 5' UTR m6A promotes Cap-independent translation [J]. Cell, 2015, 163(4): 999-1010.

[59] DOMINISSINI D, MOSHITCH-MOSHKOVITZ S, SCHWARTZ S, et al. Topology of the human and mouse m6A RNA methylomes revealed by m6A-seq[J]. Nature, 2012, 485(7397): 201-206.

[60] LIU J, YUE Y, HAN D, et al. A METTL3-METTL14 complex mediates mammalian nuclear RNA N6adenosine methylation $[\mathrm{J}]$. Nat Chem Biol, 2014, 10(2): 93-95.

[61] JIA G, FU Y, ZHAO X, et al. N6-methyladenosine in nuclear RNA is a major substrate of the obesityassociated FTO $[\mathrm{J}]$. Nat Chem Biol, 2011, 7(12): 885-887.

[62] ZHENG G, DAHL J A, NIU Y, et al. ALKBH5 is a mammalian RNA demethylase that impacts RNA metabolism and mouse fertility $[\mathrm{J}]$. Mol Cell, 2013,
49(1): 18-29.

[63 ] WANG X, ZHAO B S, ROUNDTREE I A, et al. N6methyladenosine modulates messenger RNA translation efficiency[J]. Cell, 2015, 161(6): 1388-1399.

[64] FRYE M, JAFFREY S R, PAN T, et al. RNA modifications: what have we learned and where are we headed? $[J]$. Nat Rev Genet, 2016, 17(6): 365-372.

[65] FU Y, DOMINISSINI D, RECHAVI G, et al. Gene expression regulation mediated through reversible m6A RNA methylation[J]. Nat Rev Genet, 2014, 15(5): 293-306.

[66] BATISTA P J. The RNA modification N6-methyladenosine and its implications in human disease $[\mathrm{J}]$. Genomics Proteomics BioInf, 2017, 15(3): 154-163.

[67] SHAFIK A M, ZHANG F, GUO Z, et al. N6methyladenosine dynamics in neurodevelopment and aging, and its potential role in Alzheimer's disease $[\mathrm{J}]$. Genome Biol, 2021, 22(1): 17.

[68] HAN M, LIU Z, XU Y, et al. Abnormality of m6A mRNA methylation is involved in Alzheimer's disease $[$ J ]. Front Neurosci, 2020, 14: 98.

[69] FOO J N, TAN L C, IRWAN I D, et al. Genome-wide association study of Parkinson's disease in east Asians[J]. Hum Mol Genet, 2017, 26(1): 226-232.

[70 ] CHEN X, YU C, GUO M, et al. Down-regulation of m6A mRNA methylation is involved in dopaminergic neuronal death $[\mathrm{J}]$. ACS Chem Neurosci, 2019, 10(5): 2355-2363.

[本文编辑 李 璐 沈 敏]

\section{杜永忠教授团队提出肿瘤靶向控释化疗与免疫协同治疗新策略}

2021年8月6日,浙江大学药学院杜永忠教授团队在《自然 ·通讯》(Nature Communications)在线发表了题为 "Synergistic effect of tumor chemo-immunotherapy induced by leukocyte-hitchhiking thermal-sensitive micelles" 的研究论文 (https://www. nature. com/articles/s41467-021-24902-2)。一种通过 “搭乘白细胞便车” 实现肿瘤高 效富集的温敏性载药胶束在局部微波热疗的刺激下可显著提高肿瘤免疫原性,同时有效缓解肿瘤免疫抑制微环境。

研究人员设计了一种共载免疫原性细胞死亡诱导剂阿霉素和腺苷A2A受体(A2AR)拮抗剂SCH 58261的温敏性胶 束,并在其表面修饰 $\mathrm{E}$-选择素, 静脉注射后其在 $\mathrm{E}$-选择素的介导下黏附在白细胞的表面,随同迁移至肿瘤部位,实现纳米 粒的肿瘤高度富集。积累在肿瘤部位的载药胶束在局部微波热疗刺激下实现药物的快速释放,所释放的阿霉素在阻滞 肿瘤细胞周期的同时还可以诱导肿瘤发生免疫原性细胞死亡,激活机体的肿瘤特异性细胞免疫; 而SCH 58261通过有效 拮抗各种免疫细胞表面的A2AR, 缓解肿瘤免疫抑制微环境,最终实现化疗和免疫治疗的协同抗肿瘤作用。

祁菁和金斐昒博士研究生为论文第一作者。研究得到国家重点研发计划和浙江省自然科学基金等支持。 